\title{
Peripheral arterial disease in the elderly
}

\author{
Wilbert S Aronow \\ Cardiology Division, Department \\ of Medicine, New York Medical \\ College, Valhalla, New York
}

\begin{abstract}
Smoking should be stopped and hypertension, diabetes mellitus, dyslipidemia, and hypothyroidism treated in elderly patients with peripheral arterial disease (PAD) of the lower extremities. Statins reduce the incidence of intermittent claudication and improve exercise duration until the onset of intermittent claudication in patients with PAD and hypercholesterolemia. Antiplatelet drugs such as aspirin or clopidogrel, especially clopidogrel, angiotensin-converting enzyme inhibitors, and statins should be given to all elderly patients with PAD without contraindications to these drugs. Beta blockers should be given if coronary artery disease is present. Exercise rehabilitation programs and cilostazol increase exercise time until intermittent claudication develops. Chelation therapy should be avoided. Indications for lower extremity percutaneous transluminal angioplasty or bypass surgery are (1) incapacitating claudication in patients interfering with work or lifestyle; (2) limb salvage in patients with limb-threatening ischemia as manifested by rest pain, nonhealing ulcers, and/or infection or gangrene; and (3) vasculogenic impotence.
\end{abstract}

Keywords: peripheral arterial disease, intermittent claudication, antiplatelet drugs, statins, angiotensin-converting enzyme inhibitors, cilostazol, exercise rehabilitation, revascularization

\section{Introduction}

Peripheral arterial disease (PAD) is chronic arterial occlusive disease of the lower extremities caused by atherosclerosis. PAD may cause intermittent claudication which is pain or weakness with walking that is relieved with rest. The muscle pain or weakness after exercise occurs distal to the arterial obstruction. Since the superficial femoral and popliteal arteries are most commonly affected by atherosclerosis, the pain of intermittent claudication is most commonly localized to the calf. Atherosclerotic obstruction of the distal aorta and its bifurcation into the two iliac arteries may cause pain in the buttocks, hips, thighs, or the inferior back muscles as well as the calves.

The Rutherford classification of PAD includes 7 stages (Dormandy et al 2000). PAD is classified as stage 0 if the person is asymptomatic, stage 1 if mild intermittent claudication is present, stage 2 if moderate intermittent claudication is present, stage 3 if severe intermittent claudication is present, stage 4 if ischemic rest pain is present, stage 5 if the person has minor tissue loss, and stage 6 if the person has ulceration or gangrene.

Only one-half of elderly persons with documented PAD are symptomatic. Persons with PAD may not walk far or fast enough to induce muscle ischemic symptoms because of comorbidities such as pulmonary disease or arthritis, may have atypical symptoms unrecognized as intermittent claudication, may fail to mention their symptoms to their physician, or may have sufficient collateral arterial channels to tolerate their arterial obstruction. Women with PAD have a higher prevalence of leg pain on exertion and at rest, poorer functioning, and greater walking impairment from leg symptoms than men with PAD (McDermott et al 2003a). Poorer leg strength in women contributes to poorer lower extremity functioning in women with PAD than 
in men with PAD. Upper extremity PAD will cause unequal blood pressure measurements in each arm. PAD patients also have a higher prevalence of cognitive impairment and erectile dysfunction.

If the arterial flow to the lower extremities cannot meet the needs of resting tissue metabolism, critical lower extremity ischemia occurs with pain at rest or tissue loss. Critical ischemia causes rest pain in the toes or foot with progression to ulceration or gangrene. Chronic arterial insufficiency ulcers commonly develop at the ankle, heel, or leg. Mummified, dry, black toes or devitalized soft tissue covered by a crust is gangrene caused by ischemic infarction. Suppuration often develops with time, and dry gangrene changes to wet gangrene.

\section{Physical examination}

The vascular physical examination includes (1) measurement of the blood pressure in both arms, (2) palpation of the carotid pulses and listening for carotid bruits, (3) auscultation of the abdomen and flank for bruits, (4) palpation of the abdomen and notation of the presence of the aortic pulsation and its maximal diameter, (5) palpation of pulses at the brachial, radial, ulnar, femoral, popliteal, dorsalis pedis, and posterior tibial sites, and (6) auscultation of both femoral arteries for femoral bruits (Hirsch et al 2006).

The shoes and socks should be removed and the feet inspected. The color, temperature, and integrity of the skin should be evaluated and the presence of distal hair loss, trophic skin changes, hypertrophic nails, and ulcerations noted (Hirsch et al 2006).

\section{Noninvasive diagnosis}

Persons with PAD of the lower extremities have reduced or absent arterial pulses. Noninvasive tests used to assess lower extremity arterial blood flow include measurement of ankle and brachial artery systolic blood pressures, characterization of velocity wave form, and duplex ultrasonography. Measurement of ankle and brachial artery systolic blood pressures using a Doppler stethoscope and blood pressure cuffs allows calculation of the ankle-brachial index (ABI) which is normally $0.9-1.2$. An ABI of $<0.90$ is $95 \%$ sensitive and $99 \%$ specific for the diagnosis of PAD (McDermott et al 2002). The lower the ABI, the more severe the restriction of arterial blood flow, and the more serious the ischemia. ABIs of $0.6-0.9$ usually correlate with mild to moderate intermittent claudication. ABIs of 0.4-0.6 usually correlate with severe intermittent claudication. With ABIs between 0.25 and 0.4 , rest pain and tissue loss are often found. Patients with calcified arteries from diabetes mellitus or renal failure occasionally have relatively non-compressible arteries leading to falsely elevated ABI values in the normal range.

In addition to measuring arterial pressure in non-palpable arteries, Doppler ultrasound methods allow characterization of the flow versus time velocity waveform. Finding biphasic flow at the groin or monophasic flow more distally is evidence of arterial obstruction even when ABI measurements are falsely increased to normal levels because of calcification.

Duplex ultrasonography combines Doppler frequency measurements with two-dimensional images of blood vessels. The severity of flow restriction caused by an arterial stenosis can be accurately assessed by this most comprehensive noninvasive method (Kohler et al 1987). Dupex ultrasonography, computed tomographic angiography, and magnetic resonance angiography are useful in assessing the anatomic location and severity of PAD and in selecting suitable candidates for endovascular or surgical revascularization (Hirsch et al 2006).

Treadmill exercise testing with and without pre-exercise and postexercise ABIs helps differentiate claudication from pseudoclaudication in patients with exertional leg symptoms (Hirsch et al 2006). Treadmill exercise testing may be useful to diagnose PAD with a normal resting $\mathrm{ABI}$ but a reduced postexercise ABI. Treadmill exercise testing may objectively document the magnitude of symptom limitation in patients with claudication.

\section{Prevalence}

The prevalence of PAD increases with age (Criqui et al 1985; Newman et al 1993; Meijer et al 1998; Ness et al 2000; Hirsch et al 2001; Aronow et al 2002c; Ness et al 2005;). The prevalence of symptomatic PAD was $32 \%$ in 1160 men, mean age 80 years, and $26 \%$ in 2464 women, mean age 81 year, living in a nursing home (Aronow et al 2002c). The prevalence of PAD in 6979 men and women, mean age 69 years, screened for PAD by an ABI because they were aged 70 years or older or because they were aged 50-69 years with a history of cigarette smoking or diabetes mellitus was 29\% (Hirsch et al 2001). Among these patients with PAD, classic claudication was present in only $11 \%$.

\section{Risk factors}

Modifiable risk factors that predispose to PAD include cigarette smoking (Pomrehn et al 1986; Stokes et al 1987; Aronow et al 1988; Ness et al 2000; Sukhija et al 2003; Ness et al 2005), diabetes mellitus (Pomrehn et al 1986; Stokes et al 1987; Aronow et al 1988; Ness et al 2000; 
Sukhija et al 2003; Ness et al 2005), hypertension (Ness et al 2000; Ness et al 2005; Stokes et al 1987; Aronow et al 1988; Sukhija et al 2003), dyslipidemia (Pomrehn et al 1986; Stokes et al 1987; Aronow et al 1994a; Ness et al 2000; Sukhija et al 2003; Ness et al 2005), increased plasma homocysteine levels (Malinow et al 1989; Aronow et al 1998), and hypothyroidism (Mya et al 2003). Significant independent risk factors for PAD in 467 men, mean age 80 years, and in 1444 women, mean age 81 years, living in the community and seen in an academic geriatrics paractice were age (odds ratio $=1.05$ for each 1 -year increase in age in men and 1.03 for each 1-year increase in age in women); current cigarette smoking (odds ratio $=2.6$ for men and 4.6 for women); systolic or diastolic hypertension (odds ratio $=2.2$ for men and 2.8 for women); diabetes mellitus (odds ratio $=6.1$ for men and 3.6 for women); serum high-density lipoprotein cholesterol (odds ratio $=0.95$ for each $1 \mathrm{mg} / \mathrm{dL}$ increase in men and 0.97 for each $1 \mathrm{mg} / \mathrm{dL}$ increase in women); and serum low-density lipoprotein (LDL) cholesterol (odds ratio $=1.02$ for each $1 \mathrm{mg} / \mathrm{dL}$ increase in men and in women) (Ness et al 2000).

In 147 men and women with PAD and 373 men and women without PAD, mean age 81 years, plasma homocysteine was a significant independent risk factor for PAD with an odds ratio of 1.13 for each $1 \mu \mathrm{mol} / \mathrm{L}$ increase (Aronow et al 1998). In 249 men and women, mean age 79 years, the prevalence of PAD was significantly higher in persons with subclinical hypothyroidism (14 of 18 persons or $78 \%$ ) than in persons with euthyroidism (40 of 231 persons or 17\%) (Mya et al 2003).

\section{Coexistence of other atherosclerotic disorders}

PAD coexists with other atherosclerotic disorders (Aronow et al 1994b; Ness et al 1999; Sukhija et al 2003; Ness et al 2005; Sukhija et al 2005). In a study of 1886 men and women, mean age 81 years, 270 of 468 persons (58\%) with PAD had coexistent coronary artery disease (CAD) and 159 of 468 persons (34\%) with PAD had prior ischemic stroke (Aronow et al 1994b). In a study of 1,802 men and women, mean age 80 years, living in the community and seen in an academic geriatrics practice, 161 of 236 persons (68\%) with PAD had coexistent CAD and 100 of 236 persons (42\%) with PAD had coexistent prior ischemic stroke (Ness et al 1999).

In 1,006 men and women, mean age 72 years, if PAD was present, $63 \%$ had coexistent $\mathrm{CAD}$, and $43 \%$ had prior ischemic stroke (Ness et al 2005). In 273 patients, mean age 71 years, with $\mathrm{CAD}$, the lower the $\mathrm{ABI}$, the higher the prevalence of 3-vessel or 4-vessel CAD (Sukhija et al 2005)

\section{Cardiovascular mortality and morbidity}

Persons with PAD are at increased risk for all-cause mortality, cardiovascular mortality, and cardiovascular events (Smith et al 1990; Criqui et al 1992; Aronow et al 1992; Vogt et al 1993; Newman et al 1997; Saw et al 2006). At 10-year follow-up of 565 men and women, mean age 66 years, PAD significantly increased the risk of all-cause mortality (relative risk $=3.1$ ), of mortality from cardiovascular disease (relative risk $=5.9$ ), and of mortality from CAD (relative risk $=6.6$ ) (Criqui et al 1992). At 4-year follow-up of 1492 women, mean age 71 years, an ABI of 0.9 or less was associated with a relative risk of 3.1 for all-cause mortality after adjustment for age, smoking, and other risk factors (Vogt et al 1993).

In a prospective study of 291 men and women, mean age 82 years, with PAD, CAD was present in 160 persons $(55 \%)$ (Aronow et al 1992). Silent myocardial ischemia detected by 24-hour ambulatory electrocardiography was present in 60 of 160 persons (38\%) with PAD and CAD and in 26 of 131 persons $(20 \%)$ with PAD and no clinically evident CAD. At 43-month follow-up, new coronary events developed in 54 of 60 persons $(90 \%)$ with $\mathrm{PAD}, \mathrm{CAD}$, and silent myocardial ischemia and in 59 of 100 persons (59\%) with PAD, CAD, and no silent myocardial ischemia (Aronow et al 1992). New coronary events also developed in 18 of 26 persons $(69 \%)$ with PAD, no CAD, and silent myocardial ischemia and in 34 of 105 persons (32\%) with PAD, no CAD, and no silent myocardial ischemia (Aronow et al 1992).

A pooled analysis of mortality in 8 large randomized percutaneous coronary intervention (PCI) trials of 19, 867 patients showed that the presence of PAD was associated with higher rates of post-PCI death and myocardial infarction (MI) (Saw et al 2006). PAD was an independent predictor of short-term and of long-term mortality.

\section{Risk factor modification}

Continuing smoking increases the risk of amputation in patients with intermittent claudication. Patency in lower extremity bypass grafts is also worse in smokers than in nonsmokers (Myers et al 1978). Smoking cessation reduces the progression of PAD to critical leg ischemia and reduces the risk of MI and death from vascular causes (Quick et al 1982). Smoking cessation programs should be strongly encouraged in persons with PAD (Table 1).

Approaches to smoking cessation include use of nicotine patches or nicotine polacrilex gum, which are available over the counter. If this therapy is unsuccessful, nicotine nasal spray or treatment with the antidepressant buproprion 
Table I Medical management of peripheral arterial disease in the elderly

I. Smoking cessation program

2. Treatment of hypertension with blood pressure reduced to $<140 / 90 \mathrm{~mm} \mathrm{Hg}$ and to $<130 / 80 \mathrm{~mm} \mathrm{Hg}$ in patients with diabetes mellitus or chronic renal insufficiency

3. Control diabetes mellitus with the hemoglobin $A_{I c}$ level reduced to $<7 \%$

4. Treat dyslipidemia and reduce serum low-density lipoprotein cholesterol to $<70 \mathrm{mg} / \mathrm{dL}$

5. Antiplatelet drug therapy with aspirin or preferably clopidogrel

6. Treatment with an angiotensin-converting enzyme inhibitor

7. Treatment with beta blockers in patients with coronary artery disease in the absence of contraindications to these drugs

8. Use of statins

9. Treatment with cilostazol in patients with intermittent claudication

10. Exercise rehabilitation program

II. Foot care

should be considered A nicotine inhaler may also be used. The dosage and duration of treatment of each of these pharmacotherapies are discussed in detail elsewhere (Frishman et al 2001). Concomitant behavioral therapy may also be needed. Repeated physician advice is very important in the treatment of smoking addiction.

Hypertension should be adequately controlled to decrease cardiovascular mortality and morbidity in persons with PAD (Chobanian et al 2003; Mehler et al 2003) (Table 1). The blood pressure should be reduced to $<140 / 90 \mathrm{~mm} \mathrm{Hg}$ and to $<130 / 80 \mathrm{~mm} \mathrm{Hg}$ in patients with diabetes mellitus or chronic renal insufficiency. In the Heart Outcomes Prevention Evaluation (HOPE) Study, 1715 persons had symptomatic $\mathrm{PAD}$, and 2118 persons had asymptomatic $\mathrm{PAD}$ with an $\mathrm{ABI}$ $<0.9$ (Ostergren et al 2004). In the HOPE Study, compared with placebo, ramipril $10 \mathrm{mg}$ daily significantly reduced cardiovascular events by $25 \%$ in persons with symptomatic PAD. In this study, ramipril reduced the absolute incidence of cardiovascular events by $5.9 \%$ in persons with asymptomatic PAD and by $2.3 \%$ in persons with a normal $\mathrm{ABI}$. In the HOPE Study, the antihypertensive properties of ramipril did not completely account for the observed risk reduction (Ostergren et al 2004).

Among persons with PAD in the Appropriate Blood Pressure Control in Diabetes trial, the incidence of cardiovascular events in persons treated with antihypertensive drug therapy with enalapril or nisoldipine was $13.6 \%$ if the mean blood pressure was reduced to $128 / 75 \mathrm{~mm} \mathrm{Hg}$ versus $38.7 \%$ if the mean blood pressure was reduced to $137 / 81 \mathrm{~mm} \mathrm{Hg}$ (Mehler et al 2003).

Elderly persons with diabetes mellitus and PAD and no CAD have a 1.5 times higher incidence of new coronary events than elderly nondiabetics with $\mathrm{PAD}$ and prior MI (Aronow et al 2003a). The higher the hemoglobin $\mathrm{A}_{1 \mathrm{c}}$ levels in patients with diabetes mellitus and PAD, the higher the prevalence of severe PAD (Aronow et al 2007). Diabetes mellitus should be treated with the hemoglobin $\mathrm{A}_{1 \mathrm{c}}$ level decreased to less than $7 \%$ to decrease the incidence of MI (Stratton et al 2000) (Table 1). The blood pressure should be reduced to less than 130/80 mm Hg in elderly persons with PAD and diabetes mellitus (Adler et al 2000; Chobanian et al 2003; Mehler et al 2003) (Table 1). Elderly diabetics with PAD should also be treated with statins (Aronow et al 2002a; Aronow 2002b; Grundy et al 2004) and the serum LDL cholesterol reduced to $<70 \mathrm{mg} / \mathrm{dL}$ (Grundy et al 2004) (Table 1).

Treatment of dyslipidemia with statins has been documented to reduce the incidence of mortality, cardiovascular events, and stroke in persons with PAD with and without CAD (Pedersen et al 1998; HPS 2002; Aronow et al 2002a). At 5-year follow-up of 4444 men and women with CAD and hypercholesterolemia in the Scandinavian Simvastatin Survival Study, compared with placebo, simvastatin significantly decreased the incidence of intermittent claudication by $38 \%$ (Pedersen et al 1998).

In a study of 264 men and 396 women, mean age 80 years, with symptomatic PAD and a serum LDL cholesterol of $125 \mathrm{mg} / \mathrm{dL}$ or higher, 318 of 660 persons ( $48 \%$ ) were treated with a statin and 342 of 660 persons (52\%) with no lipidlowering drug (Aronow et al 2002a). At 39-month followup, treatment with statins caused a significant independent reduction in the incidence of new coronary events of $58 \%$, of $52 \%$ in persons with prior MI, and of $59 \%$ in persons with no prior MI (Aronow et al 2002a).

In the Heart Protection Study (HPS), 6748 of the 20536 persons (33\%) had PAD (HPS 2002). At 5-year follow-up, treatment with simvastatin $40 \mathrm{mg}$ daily caused a significant $19 \%$ relative reduction and a $6.3 \%$ absolute reduction in major cardiovascular events independent of age, gender, or serum lipids levels (HPS 2002). These data favor administration of statins to elderly persons with PAD regardless of serum lipids levels.

On the basis of the available data, elderly persons with PAD and hypercholesterolemia should be treated with statins to reduce cardiovascular mortality and morbidity and progression of PAD (Pedersen et al 1998; Aronow et al 2002a; HPS 2002) and to improve exercise time until intermittent claudication (Aronow et al 2003b; Mohler et al 2003; Mondillo et al 2003). On the basis of data from the HPS, persons with PAD should be treated with statins regardless of age, gender, or initial serum lipids levels (HPS 2002). 
Increased plasma homocysteine level is a risk factor for PAD (Malinow et al 1989; Aronow et al 1998). Reduction of increased plasma homocysteine levels can be achieved by administering a combination of folic acid, vitamin B6, and vitamin B12. However, we do not have double-blind, randomized, placebo-controlled data showing that reduction of increased plasma homocysteine levels will reduce coronary events and slow progression of PAD in elderly persons with PAD.

Hypothyroidism is a risk factor for PAD (Mya et al 2003). Elderly persons with clinical or subclinical hypothyroidism should be treated with 1-thyroxine to decrease the development of CAD (Mya et al 2002). There is no evidence showing that treatment with 1-thyroxine will reduce the development of PAD or improve symptoms.

\section{Antiplatelet drugs}

Antiplatelet drugs that have been shown to decrease the incidence of vascular death, nonfatal MI, and nonfatal stroke in persons with PAD are aspirin, ticlodipine, and clopidogrel (ATCG 2002). Aspirin plus dipyridamole has not been shown to be more efficacious than aspirin alone in the treatment of persons with PAD (ATCG 2002). Adverse hematologic effects associated with ticlodipine limit the use of this drug in the treatment of elderly persons with PAD (Bennett et al 1998).

The Antithrombotic Trialists' Collaboration Group(ATCG) reported a meta-analysis of 26 randomized studies of 6,263 persons with intermittent claudication due to PAD (ATCG 2002). At follow-up, the incidence of vascular death, nonfatal MI, and nonfatal stroke was $6.4 \%$ in patients randomized to antiplatelet drugs versus $7.9 \%$ in the control group, a significant reduction of $23 \%$ caused by antiplatelet therapy. The reductions are significant for all subgroups.

The ATCG reported a meta-analysis of 12 randomized studies of 2,497 persons with PAD undergoing peripheral arterial grafting (ATCG 2002). At follow-up, the incidence of vascular death, nonfatal MI, and nonfatal stroke was 5.4\% in persons randomized to antiplatelet drugs versus $6.5 \%$ in the control group, a significant reduction of $22 \%$ caused by antiplatelet therapy.

The ATCG also reported a meta-analysis of 4 randomized studies of 946 persons with PAD undergoing peripheral angioplasty (ATCG 2002) At follow-up, the incidence of vascular death, nonfatal MI, and nonfatal stroke was $2.5 \%$ in patients randomized to antiplatelet drugs versus $3.6 \%$ in the control group, a significant reduction of $29 \%$ caused by antiplatelet therapy.
If one combines the 42 randomized studies of 9,706 patients with intermittent claudication, peripheral arterial grafting, or peripheral angioplasty, the incidence of vascular death, nonfatal MI, and nonfatal stroke at follow-up was significantly decreased $23 \%$ by antiplatelet drugs, with similar benefits among patients with intermittent claudication, those having peripheral arterial grafting, and those having peripheral angioplasty (ATCG 2002). These data favor the use of aspirin in men and women with PAD (Table 1).

\section{Aspirin}

The ATCG showed that the efficacy of different doses of aspirin in reducing in high-risk persons the incidence of vascular death, nonfatal MI, and nonfatal stroke was 19\% for aspirin 500-1500 mg daily, 26\% for aspirin 160-325 mg daily, 32\% for aspirin 75-150 mg daily, and 13\% for aspirin $<75 \mathrm{mg}$ daily (ATCG 2002). Since aspirin doses greater than $150 \mathrm{mg}$ daily do not reduce vascular death, nonfatal MI, and nonfatal stroke more than does a dose of 75 to 150 mg daily and cause more gastrointestinal bleeding than the lower doses, this author prefers an aspirin dose of 81 mg daily in treating elderly persons with atherosclerotic vascular disease.

\section{Clopidogrel}

In the Clopidogrel versus Aspirin in Patients at Risk for Ischaemic Events (CAPRIE) trial, 5,795 persons with PAD were randomized to clopidogrel $75 \mathrm{mg}$ daily and 5797 persons with PAD were randomized to aspirin $325 \mathrm{mg}$ daily (CAPRIE 1996). At 1.9-year follow-up, the annual incidence of vascular death, nonfatal MI, and nonfatal stroke was $3.7 \%$ in persons randomized to clopidogrel versus $4.9 \%$ in persons randomized to aspirin, a $24 \%$ significant decrease with the use of clopidogrel (CAPRIE 1996). In 9478 patients with prior MI, ischemic stroke, or symptomatic PAD in the Clopidogrel for High Atherothrombotic Risk and Ischemic Stabilization, Management, and Avoidance (CHARISMA) trial, addition of clopidogrel to aspirin significantly reduced cardiovascular death, MI, or stroke by $17 \%$ (Bhatt et al 2007).

On the basis of these data, it is reasonable to conclude that clopidogrel is superior to aspirin in the management of patients with PAD. On the basis of these data, the author also recommends the use of clopidogrel $75 \mathrm{mg}$ daily in the treatment of patients with PAD (Table 1). However, clopidogrel is much more expensive than is aspirin. Clopidogrel but not aspirin is approved by the USA Food and Drug Administration for the treatment of PAD. 


\section{Oral anticoagulants}

In the Dutch Bypass Oral Anticoagulants or Aspirin Study, 2690 persons were randomized after infrainguinal bypass surgery to aspirin $80 \mathrm{mg}$ daily or to oral anticoagulation with phenprocoumon or acenocoumarol to maintain an INR of 3.0-4.5 (BOA Study group 2000). At 21-month followup, there was no significant difference between the two treatments in the primary outcome of infrainguinal graft occlusion. There was no significant difference between the two treatments in the secondary outcomes of MI, stroke, amputation, or vascular death. However, persons treated with oral anticoagulant therapy had 1.96 times more major bleeding episodes than persons treated with oral aspirin. The American College of Cardiology (ACC)/American Heart Association (AHA) guidelines state that oral anticoagulant therapy with warfarin should not be given to reduce the risk of adverse cardiovascular ischemic events in persons with atherosclerotic lower extremity PAD (Hirsch et al 2006).

\section{Angiotensin-converting enzyme inhibitors}

Data from the HOPE Study showed that ramipril $10 \mathrm{mg}$ daily significantly decreased cardiovascular events in persons with symptomatic PAD and in persons with asymptomatic PAD (Ostergren et al 2004). Angiotensin-converting enzyme inhibitors as well as statins also have many pleotropic effects to account for their vascular protective properties beyond their primary mode of action including inhibition of cellular proliferation, restoration of endothelial activity, inhibition of platelet reactivity, and an antioxidant potential (Faggioto et al 1999). The ACC/AHA guidelines recommend treating persons with PAD with angiotensin-converting enzyme inhibitors unless there are contraindications to the use of these drugs to reduce cardiovascular mortality and morbidity (Smith et al 2001) (Table 1).

\section{Beta blockers}

Persons with PAD are at increased risk for developing new coronary events (Smith et al 1990; Criqui et al 1992; Aronow et al 1992; Vogt et al 1993; Newman et al 1997; Saw et al 2006). Many physicians have been reluctant to use beta blockers in persons with PAD because of concerns that beta blockers will aggravate intermittent claudication. However, a meta-analysis of 11 randomized controlled studies found that beta blockers do not adversely effect walking capacity or the symptoms of intermittent claudication in persons with mild-to-moderate PAD (Radack et al 1991).
An observational study was performed in 575 men and women, mean age 80 years, with symptomatic PAD and prior MI (Aronow et al 2001). Of the 575 persons, 85 persons (15\%) had contraindications to the use of beta blockers. Of the 490 persons without contraindications to the use of beta blockers, 257 persons (52\%) were treated with beta blockers. Adverse effects causing cessation of beta blockers occurred in 31 of the 257 persons (12\%). At 32-month follow-up, use of beta blockers caused a $53 \%$ significant independent decrease in the incidence of new coronary events in elderly persons with PAD and prior MI (Aronow et al 2001). Beta blockers should be used to treat $\mathrm{CAD}$ in patients with PAD in the absence of contraindications to these drugs (Table 1).

\section{Statins}

On the basis of data from the HPS, persons with PAD should be treated with statins regardless of age, gender, or initial serum lipids levels (HPS 2002) (Table 1). In 6748 persons with PAD in the HPS study, simvastatin caused an absolute reduction in first major vascular event of 63 per 1000 patients treated (HPS 2007). Three double-blind, randomized, placebo-controlled studies have also demonstrated that statins improve walking performance in persons with PAD (Aronow et al 2003b; Mohler et al 2003; Mondillo et al 2003).

In a study of 69 persons, mean age 75 years, with intermittent claudication, a mean ABI of 0.63, and a serum LDL cholesterol of $125 \mathrm{mg} / \mathrm{dL}$ or higher, 3 of 34 persons (9\%) treated with simvastatin and 6 of 35 persons (17\%) treated with placebo died before the 1-year study was completed (Aronow et al 2003b). Compared with placebo, simvastatin significantly increased the treadmill exercise time until the onset of intermittent claudication by $24 \%$ at 6 months and by $42 \%$ at 1 year after therapy.

In a study of 354 persons, mean age 68 years, with intermittent claudication and hypercholesterolemia, at 1-year follow-up, compared with placebo, atorvastatin $80 \mathrm{mg}$ daily significantly improved pain-free treadmill walking distance by $40 \%$ and significantly improved communitybased physical activity (Mohler et al 2003). In a study of 86 persons, mean age 67 years, with intermittent claudication and hypercholesterolemia, at 6-month follow-up, compared with placebo, simvastatin $40 \mathrm{mg}$ daily significantly improved pain-free walking distance and total walking distance on a treadmill, significantly improved the mean $\mathrm{ABI}$ at rest and after exercise, and significantly improved symptoms of claudication (Mondillo et al 2003).

Statin use is also associated with superior leg functioning independent of cholesterol levels and other potential 
confounders (McDermott et al 2003b). The data suggest that non-cholesterol-lowering properties of statins may favorably influence functioning in persons with and without PAD (McDermott et al 2003b).

\section{Drugs to increase walking distance}

Chelation therapy has been demonstrated to be ineffective in the therapy of PAD (Ernst 1997). Numerous drugs have been shown to be ineffective in improving walking distance in persons with intermittent claudication (Hiatt 2001; Eberhardt et al 2000). Beraprost sodium, an orally active prostaglandin $\mathrm{I}_{2}$ analogue, was demonstrated to be no more effective than placebo in persons with intermittent claudication (Mohler et al 2003). Naftidrofuryl (Lehert et al 1994) and propionyl levocarnitine (Brevetti et al 1999) have been reported to improve exercise walking distance in persons with intermittent claudication but have not been approved for use in the United States.

Two drugs, pentoxifylline and cilostazol, have been approved by the United States Food and Drug Administration for symptomatic treatment of intermittent claudication. However, many studies have found no consistent improvement with pentoxifylline in patients with intermittent claudication in comparison with placebo (Porter et al 1982; Dawson et al 2000; Eberhardt et al 2000).

Cilostazol inhibits phosphodiesterase type 3, increasing intracellular concentration of cyclic adenosine monophosphate. Cilostazol suppresses platelet aggregation and also acts as a direct arterial vasodilator. Cilostazol has been found in numerous trials to improve exercise capacity in patients with intermittent claudication (Dawson et al 1998; Money et al 1998; Eberhardt et al 2000; Thompson et al 2002; Dawson et al 2000). and in a dose of $100 \mathrm{mg}$ twice daily, was shown to be superior to both placebo and pentoxifylline (Dawson et al 2000).

Cilostazol should be administered to patients with PAD to increase walking distance (Table 1) but should not be given to persons with PAD who also have heart failure. Other contraindications to the use of cilostazol include a creatinine clearance $<25 \mathrm{ml} / \mathrm{min}$, a known predisposition for bleeding, or coadministration of CYP3A4 or CYP2C19 inhibitors such as cimetidine, diltiazem, erythromycin, ketoconazole, lansoprazole, omeprazole, and HIV-1 protease inhibitors.

\section{Exercise rehabilitation}

Exercise rehabilitation programs have been documented to increase walking distance in persons with intermittent claudication through improvements in peripheral circulation, walking economy, and cardiopulmonary function (Gardner et al 2000). The optimal exercise program for improving claudication pain distance in persons with PAD uses intermittent walking to near-maximal pain during a program of at least 6 months (Gardner et al 1995). Strength training is less effective than treadmill walking (Hiatt et al 1994). The ACC/AHA guidelines recommend a supervised exercise program for patients with intermittent claudication (Hirsch et al 2006) (Table 1).

Supervised exercise training is recommended for a minimum of 30-45 minutes in sessions performed at least 3 times per week for a minimum of 12 weeks (Hirsch et al 2006) and preferably for 6 months or longer (Gardner et al 1995). Among persons with PAD, self-directed walking exercise performed at least 3 times weekly is associated with significantly less functional decline during the subsequent year (McDermott et al 2006).

\section{Foot care}

Persons with PAD must have proper foot care (Fujitani et al 2004; Hirsch et al 2006) (Table 1). They must wear properly fitted shoes. Careless nail clipping or injury from walking barefoot must be avoided. Feet should be washed daily and the skin kept moist with topical emollients to prevent cracks and fissures, which may have portals for bacterial infection. Fungal infection of the feet must be treated. Socks should be wool or other thick fabrics, and padding or shoe inserts may be used to prevent pressure sores.

\section{Lower extremity angioplasty and bypass surgery}

Indications for lower extremity percutaneous transluminal angioplasty or bypass surgery are (1) incapacitating claudication in persons interfering with work or lifestyle; (2) limb salvage in persons with limb-threatening ischemia as manifested by rest pain, nonhealing ulcers, and/or infection or gangrene; and (3) vasculogenic impotence (Weitz et al 1996). Percutaneous transluminal angioplasty can be performed if there is a skilled vascular interventionalist and the arterial disease is localized to a vessel segment less than $10 \mathrm{~cm}$ in length (Weitz et al 1996). Compared to percutaneous transluminal angioplasty alone, stenting improves 3-year patency by $26 \%$ ) (Palmaz et al 1990).

Percutaneous balloon angioplasty and/or stenting is indicated for short-segment stenoses, whereas multisegment disease and occlusions are most effectively treated with surgical revascularization (Comerota 2001). Revascularization of PAD is discussed extensively elsewhere (Hirsch et al 2006; 
Fujitani et al 2004). In patients presenting with severe limb ischemia caused by infra-inguinal disease and who are suitable for either surgery or angioplasty, by-pass surgery and balloonangioplasty are associated with similar outcomes in terms of amputation-free survival. (Basil 2005). Patients with intermittent claudication should be considered for revascularization to improve symptoms only in the absence of other disease that would limit exercise improvement such as angina pectoris, heart failure, chronic pulmonary disease, or orthopedic limitations (Hirsch et al 2006).

\section{Amputation}

Nonrandomized studies have demonstrated that both immediate and long-term survival are higher in patients having revascularization rather than amputation for limb-threatening ischemia (Ouriel et al 1988; DeFrang et al 1991). However, amputation of lower extremities should be performed if tissue loss has progressed beyond the point of salvage, if surgery is too risky, if life expectancy is very low, or if functional limitations diminish the benefit of limb salvage (Fujitani et al 2004).

\section{References}

Adler AI, Stratton IM, Neil HAW, et al. 2000. Association of systolic blood pressure with macrovascular and microvascular complications of type 2 diabetes (UKPDS 36):prospective observational study. BMJ, 321:412-19.

Antithrombotic Trialists' Collaboration. 2002. Collaborative meta-analyis of randomised trials of antiplatelet therapy for prevention of death, myocardial infarction, and stroke in high risk patients. BMJ, 324:71-86.

Aronow WS, Ahn C. 1994a. Correlation of serum lipids with the presence or absence of atherothrombotic brain infarction and peripheral arterial disease in 1,834 men and women aged $\geq 62$ years. Am J Cardiol, 73:995-7.

Aronow WS, Ahn C. 1994b. Prevalence of coexistence of coronary artery disease, peripheral arterial disease, and atherothrombotic brain infarction in men and women $\geq 62$ years of age. Am J Cardiol, 74:64-5.

Aronow WS, Ahn C. 1998. Association between plasma homocysteine and peripheral arterial disease in older persons. Coronary Artery Dis, 9:49-50.

Aronow WS, Ahn C. 2001. Effect of beta blockers on incidence of new coronary events in older persons with prior myocardial infarction and symptomatic peripheral arterial disease. Am J Cardiol, 87:1284-6.

Aronow WS, Ahn C. 2002a. Frequency of new coronary events in older persons with peripheral arterial disease and serum low-density lipoprotein cholesterol $\geq 125 \mathrm{mg} / \mathrm{dl}$ treated with statins versus no lipid-lowering drug. Am J Cardiol, 90:789-91.

Aronow WS, Ahn C. 2003a. Elderly diabetics with peripheral arterial disease and no coronary artery disease have a higher incidence of new coronary events than elderly nondiabetics with peripheral arterial disease and prior myocardial infarction treated with statins and with no lipidlowering drug. J Gerontol A Biol Sci Med Sci, 58:M573-5.

Aronow WS, Ahn C, Gutstein H. 2002b. Reduction of new coronary events and of new atherothrombotic brain infarction in older persons with diabetes mellitus, prior myocardial infarction, and serum low-density lipoprotein cholesterol $\geq 125 \mathrm{mg} / \mathrm{dL}$ treated with statins. J Gerontol A Biol Sci Med Sci, 57A:M747-50.
Aronow WS, Ahn C, Gutstein H. 2002c. Prevalence and incidence of cardiovascular disease in 1160 older men and 2464 older women in a long-term health care facility. J Gerontol A Biol Sci Med Sci, 57A: M45-6.

Aronow WS, Ahn C, Mercando AD, Epstein S. 1992. Prognostic significance of silent ischemia in elderly patients with peripheral arterial disease with and without previous myocardial infarction. Am J Cardiol, 69:137-9.

Aronow WS, Ahn C, Weiss MB, et al. 2007. Relation of increased hemoglobin $\mathrm{A}_{1 \mathrm{c}}$ levels to severity of peripheral arterial disease in patients with diabetes mellitus. Am J Cardiol, 99:1468-9.

Aronow WS, Nayak D, Woodworth S, et al. 2003b. Effect of simvastatin versus placebo on treadmill exercise time until the onset of intermittent claudication in older patients with peripheral arterial disease at 6 months and at 1 year after treatment. Am J Cardiol, 92:711-12.

Aronow WS, Sales FF, Etienne F, et al. 1988. Prevalence of peripheral arterial disease and its correlation with risk factors for peripheral arterial disease in elderly patients in a long-term health care facility. Am J Cardiol, 62:644-6.

BASIL trial participants. 2005. Bypass versus angioplasty in severe ischaemia of the leg (BASIL):multicentre, randomised controlled trial. Lancet, 366:1925-34.

Bennett CL, Weinberg PD, Rozenberg-Ben-Dror K, et al. 1998. Thrombotic thrombocytopenic purpura associated with ticlopidine; a review of 60 cases. Ann Intern Med, 128:541-4.

Bhatt DL, Flather MD, Hacke W, et al. 2007. Patients with prior myocardial infarction, stroke, or symptomatic peripheral arterial disease in the CHARISMA trial. J Am Coll Cardiol, 49:1982-8.

Brevetti G, Perna S, Sabba C, et al. 1999. Propionyl-L-carnitine in intermittent claudication: a double-blind, placebo-controlled, dose titration, multicenter study. J Am Coll Cardiol, 26:1411-16.

CAPRIE Steering Committee. 1996. A randomised, blinded, trial of clopidogrel versus aspirin in patients at risk of ischaemic events (CAPRIE). Lancet, 348:1329-39.

Chobanian AV, Bakris GL, Black HR, et al. 2003. The Seventh Report of the Joint National Committee on Prevention, Detection, Evaluation, and Treatment of High Blood Pressure:the JNC 7 report. JAMA, 289:2560-72.

Comerota AJ. 2001. Endovascular and surgical revascularization for patients with intermittent claudication. Am J Cardiol, 87(suppl):34D-43D.

Criqui MH, Fronek A, Barrett-Connor E, et al. 1985. The prevalence of peripheral arterial disease in a defined population. Circulation, 71:510-15.

Criqui MH, Langer RD, Fronek A, et al. 1992. Mortality over a period of 10 years in patients with peripheral arterial disease. $N$ Engl J Med, 326:381-6.

Dawson DL, Cutler BS, Hiatt WR, et al. 2000. A comparison of cilostazol and pentoxifylline for treating intermittent claudication. $\mathrm{Am} \mathrm{J} \mathrm{Med,}$ 109:523-30.

Dawson DL, Cutler BS, Meissner MH, et al. 1998. Cilostazol has beneficial effects in treatment of intermittent claudication. Results from a multicenter, randomized, prospective, double-blind trial. Circulation, 98:678-86.

DeFrang RD, Taylor LM Jr, Porter JM. 1991. Basic data related to amputations. Ann Vasc Surg, 5:202-7.

Dormandy JA, Rutherford RB, for the TASC Working Group.TransAtlantic Inter-Society Consensus (TASC). 2000. Management of peripheral arterial disease (PAD). J Vasc Surg, 31:S1-S296.

Dutch Bypass Oral Anticoagulants or Aspirin (BOA) Study Group. 2000. Efficacy of oral anticoagulants compared with aspirin after infrainguinal bypass surgery (The Dutch Bypass Oral Anticoagulants or Aspirin Study): a randomized trial. Lancet, 355:346-51.

Eberhardt RT, Coffman JD. 2000. Drug treatment of peripheral vascular disease. Heart Dis, 2:62-74.

Ernst E 1997. Chelation therapy for peripheral arterial occlusive disease:a systematic review. Circulation, 96:1031-3. 
Faggiotto A, Paoletti R. 1999. Statins and blockers of the renin-angiotensin system. Vascular protection beyond their primary mode of action. Hypertension, 34:987-96

Frishman WH, Ky T, Ismail A. 2001. Tobacco smoking, nicotine, and nonnicotine replacement therapies. Heart Dis, 3:365-77

Fujitani RM, Gordon IL, Perera GB, et al. 2004. Peripheral vascular disease in the elderly. In: Aronow WS, Fleg JL, eds. Cardiovascular Disease in the Elderly Patient, 3rd ed. New York City: Marcel Dekker, Inc, 707-63.

Gardner AW, Katzel LI, Sorkin JD, et al. 2000. Improved functional outcomes following exercise rehabilitation in patients with intermittent claudication. J Gerontol A Biol Sci Med Sci, 55A:M570-M57.

Gardner AW, Poehlman ET 1995. Exercise rehabilitation programs for the treatment of claudication pain. A meta-analysis. JAMA, 274:975-80.

Grundy SM, Cleeman JI, Merz CN, et al. 2004. Implications of recent clinical trials for the National Cholesterol Education Program Adult Treatment Panel III guidelines. Circulation, 110:227-39.

Heart Protection Study Collaborative Group. 2002. MRC/BHF Heart Protection Study of cholesterol lowering with simvastatin in 20,536 high-risk individuals:a randomised placebo-controlled trial. Lancet, 360:7-22.

Hiatt WR 2001. Medical treatment of peripheral arterial disease and claudication. $N$ Engl J Med, 344:1608-21.

Hiatt WR, Wolfel EE, Meier RH, et al. 1994. Superiority of treadmill walking exercise versus strength training for patients with peripheral arterial disease. Implications for the mechanism of the training response. Circulation, 90:1866-74.

Hirsch AT, Criqui MH, Treat-Jacobson D, et al. 2001. Peripheral arterial disease detection, awareness, and treatment in primary care. JAMA, 286:1317-24.

Hirsch AT, Haskal ZJ, Hertzer NR, et al. 2006. ACC/AHA 2005 Practice Guidelines for the management of patients with peripheral arterial disease (lower extremity, renal, mesenteric, and abdominal aortic): Executive Summary. Circulation, 113:1474-547.

Kohler TR, Nance DR, Cramer MM, et al. 1987. Duplex scanning for diagnosis of aortoiliac and femoropopliteal disease:a prospective study. Circulation, 76:1074-80.

Lehert P, Comte S, Gamand S, et al. 1994. Naftidrofuryl in intermittent claudication:a retrospective analysis. $J$ Cardiovasc Pharmacol, 23(suppl 3):S48-S52.

Malinow MR, Kang SS, Taylor IM, et al. 1989. Prevalence of hyperhomocyst(e)inemia in patients with peripheral arterial occlusive disease. Circulation, 79:1180-8.

McDermott MM, Greenland P, Liu K, et al. 2002. The ankle brachial index is associated with leg function and physical activity:the Walking and Leg Circulation Study. Ann Intern Med, 136:873-83

McDermott MM, Greenland P, Liu K, et al. 2003a Sex differences in peripheral arterial disease:leg symptoms and physical functioning. $J$ Am Geriatr Soc, 51:222-8.

.McDermott MM, Guralnik JM, Greenland P, et al. 2003b. Statin use and leg functioning in patients with and without lower-extremity peripheral arterial disease. Circulation, 107:757-61.

McDermott MM, Liu K, Ferrucci L, et al. 2006. Physical performance in peripheral arterial disease:a slower rate of decline in patients who walk more. Ann Intern Med, 144:10-20.

Mehler PS, Coll JR, Estacio R, et al. 2003. Intensive blood pressure control reduces the risk of cardiovascular events in patients with peripheral arterial disease and type 2 diabetes. Circulation, 107:753-6.

Meijer WT, Hoes AW, Rutgers D, et al. 1998. Peripheral arterial disease in the elderly. The Rotterdam Study. Arterioscler Thromb Vasc Biol, 18:185-92.

Mohler ER III, Hiatt WR, Creager MA, for the Study Investigators 2003. Cholesterol reduction with atorvastatin improves walking distance in patients with peripheral arterial disease. Circulation, 108:1481-6

Mohler ER III, Hiatt WR, Olin JW, et al. 2003. Treatment of intermittent claudication with beraprost sodium, an orally active prostaglandin $\mathrm{I}_{2}$ analogue. A double-blinded, randomized, controlled trial. $J$ Am Coll Cardiol, 41:1679-86.
Mondillo S, Ballo P, Barbati R, et al. 2003. Effects of simvastatin on walking performance and symptoms of intermittent claudication in hypercholesterolemic patients with peripheral vascular disease. Am J Med, 114:359-64.

Money SR, Herd JA, Isaacsohn JL, et al. 1998. Effect of cilostazol on walking distances in patients with intermittent claudication caused by peripheral vascular disease. J Vasc Surg, 27:267-274.

Mya MM, Aronow WS. 2002. Subclinical hypothyroidism is associated with coronary artery disease in older persons. J Gerontol A Biol Sci Med Sci, 57A:M658-9.

Mya MM, Aronow WS. 2003. Increased prevalence of peripheral arterial disease in older men and women with subclinical hypothyroidism. $J$ Gerontol A Biol Sci Med Sci, 58A:M68-9.

Myers KA, King RB, Scott DF, et al. 1978. The effect of smoking on the late patency of arterial reconstructions in the legs. Br J Surg, 65:267-71.

Ness J, Aronow WS 1999. Prevalence of coexistence of coronary artery disease, ischemic stroke, and peripheral arterial disease in older persons, mean age 80 years, in an academic hospital-based geriatrics practice. $J$ Am Geriatr Soc, 47:1255-6.

Ness J, Aronow WS, Ahn C. 2000. Risk factors for peripheral arterial disease in an academic hospital-based geriatrics practice. J Am Geriatr Soc, 48:312-14.

Ness J, Aronow WS, Newkirk E, et al. 2005. Prevalence of symptomatic peripheral arterial disease, modifiable risk factors, and appropriate use of drugs in the treatment of peripheral arterial disease in older persons seen in a university general medicine clinic. J Gerontol A Biol Sci Med Sci, 60A:M255-7.

Newman A, Siscovick DS, Manolio TA, et al. 1993. Ankle-arm index as a marker of atherosclerosis in the Cardiovascular Health Study. Circulation, 88:837-45.

Newman AB, Tyrrell KS, Kuller LH. 1997. Mortality over four years in SHEP participants with a low ankle-arm index. $J$ Am Geriatr Soc, 45:1472-8.

Ostergren J, Sleight P, Dagenais G, et al. 2004. Impact of ramipril in patients with evidence of clinical or subclinical peripheral arterial disease. Eur Heart $J, 25: 17-24$

Ouriel K, Fiore WM, Geary JE. 1988. Limb-threatening ischemia in the medically compromised patient:amputation or revascularization? Surgery, 104:667-72.

Palmaz JC, Garcia OJ, Schatz RA, et al. 1990. Placement of balloonexpandable intraluminal stents in iliac arteries. First 171 procedures. Radiology, 174:969.

Pedersen TR, Kjekshus J, Pyorala K, et al. 1998. Effect of simvastatin on ischemic signs and symptoms in the Scandinavian Simvastatin Survival Study (4S). Am J Cardiol, 81:333-6.

Pomrehn P, Duncan B, Weissfeld L, et al. 1986. The association of dyslipoproteinemia with symptoms and signs of peripheral arterial disease: the Lipid Research Clinics Program Prevalence Study. Circulation, 73(suppl I):I-100-I-107.

Porter JM, Cutler BS, Lee BY, et al. 1982. Pentoxifylline efficacy in the treatment of intermittent claudication:multicenter controlled doubleblind trial with objective assessment of chronic occlusive arterial disease patients. Am Heart J, 104:66-72

Quick CRG, Cotton LT. 1982. The measured effect of stopping smoking on intermittent claudication. Br J Surg, 69(suppl):S24-6.

Radack K, Deck C. 1991. Beta-aderenergic blocker therapy does not worsen intermittent claudication in subjects with peripheral arterial disease:meta-analysis of randomized controlled trials. Arch Intern Med, 151:1769-76.

Saw J, Bhatt DL, Moliterno DJ, et al. 2006. The influence of peripheral arterial disease on outcomes. A pooled analysis of mortality in eight large randomized percutaneous coronary intervention trials. $J$ Am Coll Cardiol, 48:1567-72.

Smith SC Jr, Blair SN, Bonow RO, et al. 2001. A statement for healthcare professionals from the American Heart Association and the American College of Cardiology. J Am Coll Cardiol, 38:1581-3. 
Smith GD, Shipley MJ, Rose G. 1990. Intermittent claudication, heart disease risk factors, and mortality:the Whitehall study. Circulation, 82:1925-31.

Stokes J III, Kannel WB, Wolf PA, et al. 1987. The relative importance of selected risk factors for various manifestations of cardiovascular disease among men and women from 35 to 64 years old:30 years of follow-up in the Framingham Study. Circulation, 75(suppl V):V-65-V-73.

Stratton IM, Adler AI, Neil HAW, et al. 2000. Association of glycaemia with macrovascular and microvascular complications of type 2 diabetes (UKPDS 35):prospective observational study. BMJ, 321:405-12.

Sukhija R, Aronow WS, Yalamanchili K, et al. 2005. Association of anklebrachial index with severity of angiographic coronary artery disease in patients with peripheral arterial disease and coronary artery disease. Cardiology, 103:158-60.
Sukhija R, Yalamanchili K, Aronow WS. 2003. Prevalence of left main coronary artery disease, of 3-vessel or 4-vessel coronary artery disease, and of obstructive coronary artery disease in patients with and without peripheral arterial disease undergoing coronary angiography for suspected coronary artery disease. Am J Cardiol, 92:304-5.

Thompson PD, Zimet R, Forbes WP, et al. 2002. Meta-analysis of results from eight randomized, placebo-controlled trials on the effect of cilostazol on patients with intermittent claudication. Am J Cardiol, 90:1314-19.

Vogt MT, Cauley JA, Newman AB, et al. 1993. Decreased ankle/arm blood pressure index and mortality in elderly women. JAMA, 270:465-9.

Weitz JI, Byrne J, Clagett GP, et al. 1996. Diagnosis and treatment of chronic arterial insufficiency of the lower extremities:a critical review. Circulation, 94:3026-49. 